\title{
Multicode based communications in Impulse Radio UWB systems
}

\author{
Eric A. Bouton ${ }^{1}$, Philippe Ciblat ${ }^{1}$ and Christophe J. Le Martret ${ }^{2}$ \\ ${ }^{1}$ TELECOM ParisTech, Paris, 46, rue Barrault, 75013 Paris, France \\ Email: eric.bouton@telecom-paristech.fr, philippe.ciblat@telecom-paristech.fr \\ 2 THALES Land and Joint Systems, 160 Bd de Valmy, B.P. 82, 92704 Colombes, France \\ Email: christophe.le_martret@fr.thalesgroup.com
}

\begin{abstract}
In order to boost the data-rate in Impulse Radio UWB systems (which is necessary in some applications such as video stream), we propose to allocate more than one time-hopping code to the same user. In the case of a rake receiver, a new kind of interference associated with extra codes allocated to the user of interest is induced. Consequently the impact of these extra codes on the data rate is not straightforward. In order to evaluate the benefit of our proposition, we derive a closed-form for the multicode interference variance in the context of a time-hopping ultra wideband impulse radio based system. This enables us to theoretically analyze the influence of the number of additional codes on the real achievable data rate.
\end{abstract}

\section{INTRODUCTION}

For several years, Time-Hopping Ultra WideBand Impulse Radio (TH UWB-IR) based communication systems have received great attention, especially for short range and high data rate communication schemes [1], [2].

As a low-complexity and low-cost terminal is recommended, it is common to consider a rake receiver structure. Consequently, the performance is quite poor and the data rate is thus limited. One way to increase the data rate is to provide several channels of communication to the same user. Therefore we propose to allocate several time-hopping codes to the user who requires high data rate. If rake reception is carried out, these additional codes will unfortunately generate interference disturbing the data detection of the user of interest. Therefore, the data rate will not increase linearly with the number of allocated codes. In order to analyze the influence of the number of additional codes on the data rate, we first suggest to derive the variance of the multicode interference in a closed-form expression. Thanks to the analytical evaluation of the variance, we secondly provide a data rate approximation with respect to the number of additional time-hopping codes. Moreover, the provided closed-form expression of the variance of the multicode interference can then be studied with respect to either the number of pulses per symbol, or the statistical parameters of the propagation channel.

This work was prepared through collaborative participation in "RISC Réseaux hétérogènes intelligents pour situations de crise" project, sponsored by Agence Nationale de la Recherche (ANR) on the one hand, and through the European Union Programme of High Level Scholarships for Latin America (Alban program) under scholarship no. E06D101464BR, on the other hand.
Thus, the main purpose of this paper is to obtain a closedform expression of the multicode interference (MCI) variance at the output of a rake receiver in the context of Time-Hopping Impulse Radio UltraWide Band.

The paper is organized as follows: in Section II, we introduce the TH-IR-UWB based system as well as the propagation channel model. In Section III, we provide the original closedform expression of the MCI variance. Section IV is devoted to simulations. Conclusions are drawn in Section V.

\section{SySTEM MODEL}

For the sake of simplicity, we consider the single user case. A Pulse Amplitude Modulation (PAM) format is hereafter considered. Nevertheless, an extension of the proposed work to pulse position modulation (PPM) can be done similarly.

As a multicode based communication is considered for the unique active user, the transmit signal of this user takes the following form

$$
x(t)=\sum_{n=1}^{K} x_{n}(t)
$$

where $x_{n}(t)$ corresponds to the signal associated with the $n^{\text {th }}$ code. So, we have

$$
x_{n}(t)=\sum_{i=-\infty}^{+\infty} d_{n}(i) \sum_{j=0}^{N_{c} N_{f}-1} c_{n}(j) w\left(t-i N_{f} T_{f}-j T_{c}\right)
$$

where $N_{c}$ is the number of chips of duration $T_{c}, N_{f}$ is the number of frames of duration $T_{f}:=N_{c} T_{c}, w(t)$ is the pulse of duration $T_{w} \ll T_{c}$ [1]. The transmitted symbols $d_{n}(i) \in\{-1,1\}$ are assumed to be independent and identically distributed (i.i.d.). The so-called developed time-hopping code $\left\{c_{n}(j)\right\}_{j=0}^{N_{c} N_{f}-1}$ is defined as follows: $c_{n}(j)$ is non-null if and only if the $j^{\text {th }}$ chip associated with the $n^{\text {th }}$ way of communication is occupied by a pulse [3].

Thus, after propagation through the multipath channel, the received signal can be expressed as follows:

$$
y(t)=\sum_{k=1}^{N_{p}} A_{k} x\left(t-\tau_{k}\right)+n(t),
$$


where $A_{k}$ and $\tau_{k}$ are the amplitude and the delay of the $k^{\text {th }}$ path respectively, and where $N_{p}$ is the number of paths and $n(t)$ is a zero-mean white Gaussian noise.

The channel model considered here is the conventional one established for UWB personal area networks [5], [6], with one cluster. The amplitudes are zero-mean random variables given by $A_{k}=a_{k} \cdot e^{-\tau_{k} / 2 \gamma}$ with $\gamma$ the ray decay factor, and $a_{k}=$ $p_{k} \cdot \beta_{k}$ where $p_{k} \in\{-1,+1\}$ is an equi-likely binary random sequence and where $\beta_{k}$ is a log-normal random variable. The delays $\tau_{k}$ are independent Poisson random variables with parameter $\lambda$ and as a consequence, the difference between two consecutive delays obeys an exponential distribution with parameter $\lambda$. We define $\sigma_{a}^{2}:=\mathbb{E}_{a}\left[a_{k}^{2}\right]$, and the fourth-moment $\mu_{a}^{4}:=\mathbb{E}_{a}\left[a_{k}^{4}\right]$. We also put $I_{k}:=\mathbb{E}_{a}\left[A_{k}^{2}\right]=\sigma_{a}^{2} \cdot e^{-\tau_{k} / \gamma}$.

We consider a rake receiver that selects any subset $\mathscr{L}$ of the $L_{r}$ paths (with $L_{r} \leq N_{p}$ ). Without loss of generality, the receiver wishes to retrieve the first symbol of the first way, $d_{1}(0)$ (which is assumed to be equal to 1 ). Then, the signal at the output of the rake receiver can be written as

$$
z=\sum_{\ell \in \mathscr{L}} A_{\ell} \int_{0}^{N_{f} T_{f}} y\left(t+\tau_{\ell}\right) v_{1}(t) \mathrm{d} t
$$

where $v_{1}(t):=\sum_{j=0}^{N_{c} N_{f}-1} c_{1}(j) w\left(t-j T_{c}\right)$ is the receiver template associated with the first way of communication (i.e., associated with the first time-hopping code). By putting Eq. (1) into Eq. (2), after straightforward algebraic manipulations, we obtain

$$
z=\sum_{n=1}^{K} \sum_{\ell \in \mathscr{L}} \sum_{k=1}^{N_{p}} A_{\ell} A_{k} p_{k, \ell, n}+\eta
$$

where

$p_{k, \ell, n}=\sum_{i=-\infty}^{+\infty} d_{n}(i) \sum_{j, j^{\prime}=0}^{N_{c} N_{f}-1} c_{1}(j) c_{n}\left(j^{\prime}\right) r\left(\Delta \tau_{k, \ell}+\left(j-j^{\prime}\right) T_{c}+i N_{f} T_{f}\right)$

with $r(s):=\int_{-\infty}^{+\infty} w(t) w(t-s) d t, \Delta \tau_{k, \ell}:=\tau_{k}-\tau_{\ell}$, and $\eta$ the filtered noise due to $n(t)$ contribution.

Moreover, according to [3] and [4], the term $p_{k, \ell, n}$ can be simplified as follows:

$$
\begin{aligned}
p_{k, \ell, n} & =d_{n}\left(-Q^{k, \ell}\right)\left[\mathcal{C}_{1, n}^{+}\left(q^{k, \ell}\right) r\left(\varepsilon^{k, \ell}\right)\right. \\
& \left.+\mathcal{C}_{1, n}^{+}\left(q^{k, \ell}+1\right) r\left(\varepsilon^{k, \ell}-T_{c}\right)\right] \\
& +d_{n}\left(-Q^{k, \ell}-1\right)\left[\mathcal{C}_{1, n}^{-}\left(q^{k, \ell}\right) r\left(\varepsilon^{k, \ell}\right)\right. \\
& \left.+\mathcal{C}_{1, n}^{-}\left(q^{k, \ell}+1\right) r\left(\varepsilon^{k, \ell}-T_{c}\right)\right]
\end{aligned}
$$

where

$$
\begin{aligned}
\mathcal{C}_{1, n}^{+}(q) & :=\sum_{k=q}^{N_{c} N_{f}-1} c_{1}(k) c_{n}(k-q) \\
\mathcal{C}_{1, n}^{-}(q) & :=\sum_{k=0}^{q-1} c_{1}(k) c_{n}(k-q)
\end{aligned}
$$

and where the difference between two delays can be decomposed as follows

$$
\Delta \tau_{k, \ell}=Q^{k, \ell} N_{f} T_{f}+q^{k, \ell} T_{c}+\varepsilon^{k, \ell}
$$

with $Q^{k, \ell}=\left\lfloor\left(\Delta \tau_{k, \ell}\right) / N_{f} T_{f}\right\rfloor, q^{k, \ell}=\left\lfloor\left(\Delta \tau_{k, \ell}-\right.\right.$ $\left.\left.Q^{k, \ell} N_{f} T_{f}\right) / T_{c}\right\rfloor$ and the remainder $\varepsilon^{k, \ell} \in\left[0, T_{c}\right)$. Notice that $\lfloor x\rfloor$ is the integer-floor of $x$.

The term $z$ can be split into two terms $z=z_{u}+z_{m c i}$ with

$$
\begin{aligned}
z_{u} & :=\sum_{\ell \in \mathscr{L}} \sum_{k=1}^{N_{p}} A_{k} A_{\ell} y_{k, \ell, 1} \\
z_{m c i} & :=\sum_{n=2}^{K} \sum_{\ell \in \mathscr{L}} \sum_{k=1}^{N_{p}} A_{k} A_{\ell} y_{k, \ell, n} .
\end{aligned}
$$

The terms $z_{u}$ and $z_{m c i}$ can be interpreted as follows

- $z_{u}$ corresponds to the signal associated with the code of interest. In this term, it remains inter-symbol, inter-frame, and inter-pulse interferences which can be analysed and treated according to [7] and [8].

- $z_{m c i}$ is the multicode interference (MCI) which is a disturbing term when a decision is needed for data carried by the code of interest.

The main purpose of this paper is to derive the averaged variance of the MCI denoted by

$$
V:=\mathbb{E}_{a, d, \tau}\left[z_{m c i}^{2}\right]
$$

in closed-form expression.

\section{CLOSED-FORM EXPRESSION FOR THE MCI VARIANCE}

The derivation of the MCI variance is split into three main steps: first of all, we average the term $z_{m c i}^{2}$ over the amplitudes $a_{k}$ given the symbols $d_{n}(i)$ and the delays $\tau_{k}$. Since the amplitudes $a_{k}$ are zero-mean and independent, we get

$$
\begin{aligned}
\mathbb{E}_{a}\left[z_{m c i}^{2}\right] & =\sum_{n_{1}, n_{2}=2}^{K} \sum_{\ell \in \mathcal{L}}\left(\kappa^{\ell} y_{\ell, \ell, n_{1}} y_{\ell, \ell, n_{2}}+\sum_{k=1}^{N_{p}} I^{\ell} I^{k}\left[y_{k, \ell, n_{1}} y_{k, \ell, n_{2}}\right.\right. \\
& \left.\left.+y_{k, k, n_{1}} y_{\ell, \ell, n_{2}} \mathbf{1}_{k \in \mathcal{L}}+y_{k, \ell, n_{1}} y_{\ell, k, n_{2}} \mathbf{1}_{k \in \mathcal{L}}\right]\right)
\end{aligned}
$$

where $\mathbf{1}_{k \in \mathscr{L}}$ is equal to 1 when $k \in \mathscr{L}$ and 0 otherwise, and where $\kappa^{\ell}=\operatorname{cum}\left(A_{\ell}, A_{\ell}, A_{\ell}, A_{\ell}\right)$.

Secondly, we average Eq. (3) over the symbols $d_{n}(i)$ given the delays $\tau_{k}$. As the symbols $d_{n}(i)$ are i.i.d., we obtain

$$
\begin{aligned}
\mathbb{E}_{a, d}\left[z_{m c i}^{2}\right] & =\sum_{n=2}^{K} \sum_{\ell \in \mathcal{L}} \sum_{k=1}^{N_{p}}\left[a_{k, \ell} r^{2}\left(\varepsilon^{k, \ell}\right)\right. \\
& \left.+b_{k, \ell} r^{2}\left(\varepsilon^{k, \ell}-T_{c}\right)+c_{k, \ell} r^{2}(0)\right]
\end{aligned}
$$

where

$$
\begin{aligned}
a_{k, \ell} & =I^{\ell} I^{k}\left[\mathcal{C}_{1, n}^{+2}\left(q^{k, \ell}\right)+\mathcal{C}_{1, n}^{-2}\left(q^{k, \ell}\right)\right. \\
& +\delta_{Q^{k, \ell}, 0} \mathcal{C}_{1, n}^{+}\left(q^{k, \ell}\right) \mathcal{C}_{n, 1}^{+}\left(q^{k, \ell}\right) \mathbf{1}_{k \in \mathcal{L}} \\
& \left.+\delta_{Q^{k, \ell}+1,0} \mathcal{C}_{1, n}^{-}\left(q^{k, \ell}\right) \mathcal{C}_{n, 1}^{-}\left(q^{k, \ell}\right) \mathbf{1}_{k \in \mathcal{L}}\right] \\
b_{k, \ell} & =I^{\ell} I^{k}\left[\mathcal{C}_{1, n}^{+2}\left(q^{k, \ell}+1\right)+\mathcal{C}_{1, n}^{-2}\left(q^{k, \ell}+1\right)\right. \\
& +\delta_{Q^{k, \ell}, 0} \mathcal{C}_{1, n}^{+}\left(q^{k, \ell}+1\right) \mathcal{C}_{n, 1}^{+}\left(q^{k, \ell}+1\right) \mathbf{1}_{k \in \mathcal{L}} \\
& \left.+\delta_{Q^{k, \ell}+1,0} \mathcal{C}_{1, n}^{-}\left(q^{k, \ell}+1\right) \mathcal{C}_{n, 1}^{-}\left(q^{k, \ell}+1\right) \mathbf{1}_{k \in \mathcal{L}}\right] \\
c_{k, \ell} & =\left(\kappa^{\ell} \delta_{k, 1}+I^{\ell} I^{k} \mathbf{1}_{k \in \mathcal{L}}\right)\left(\mathcal{C}_{1, n}^{+2}(0)+\mathcal{C}_{1, n}^{-2}(0)\right)
\end{aligned}
$$

and $\delta_{m, n}$ is the so-called Kronecker index. 
We thirdly average Eq. (4) over the delays $\tau_{k}$. From now on, we only consider the partial rake receiver, i.e., $\mathscr{L}=$ $\left\{1,2, \cdots, L_{r}\right\}$. Notice that the partial rake receiver fingers are associated with the first successive delays and not with the most powerful delays as done for the so-called selective rake receiver. Unlike the selective rake receiver, the choice of the partial rake receiver enables us to derive a closedform expression for the statistics of the encountered delays and consequently, for $\mathbb{E}_{a, d, \tau}\left[z_{m c i}^{2}\right]$.

From Eq. (4), after tedious but easy algebraic manipulations, we deduce that

$$
\begin{aligned}
\mathbb{E}_{a, d, \tau}\left[z_{m c i}^{2}\right] & =\sum_{n=2}^{K} \sum_{\ell=1}^{L_{r}} \sum_{k=1}^{L_{r}}\left(\kappa_{a} M_{\ell} \delta_{k, 1}+\sigma_{a}^{4} M_{\ell, k}\right) \\
& \times\left(\mathcal{C}_{1, n}^{+2}(0)+\mathcal{C}_{1, n}^{-2}(0)\right) r^{2}(0)+\sigma_{a}^{4} M_{\ell} \\
& \times\left(\mathcal{C}_{1, n}^{+2}(0)+\mathcal{C}_{1, n}^{-2}(0)+\mathcal{C}_{1, n}^{+}(0) \mathcal{C}_{n, 1}^{+}(0)\right) r^{2}(0) \\
& +\sigma_{a}^{4} \sum_{n=2}^{K} \sum_{\ell=1}^{L_{r}} \sum_{k=\ell+1}^{L_{r}}\left(Y_{1}^{k, \ell}+Z_{1}^{k, \ell}\right) \\
& +\sigma_{a}^{4} \sum_{n=2}^{K} \sum_{\ell=1}^{L_{r}} \sum_{k=L_{r}+1}^{N_{p}}\left(Y_{2}^{k, \ell}+Z_{2}^{k, \ell}\right)
\end{aligned}
$$

with

$$
\begin{aligned}
Y_{m}^{k, \ell} & =\mathbb{E}_{\tau}\left[e^{-\left(\tau_{\ell}+\tau_{k}\right) / \gamma} G_{m}\left(Q^{k, \ell}, q^{k, \ell}\right) r^{2}\left(\varepsilon^{k, \ell}\right)\right] \\
Z_{m}^{k, \ell} & =\mathbb{E}_{\tau}\left[e^{-\left(\tau_{\ell}+\tau_{k}\right) / \gamma} J_{m}\left(Q^{k, \ell}, q^{k, \ell}\right) r^{2}\left(\varepsilon^{k, \ell}-T_{c}\right)\right.
\end{aligned}
$$

and

$$
\begin{aligned}
G_{1}\left(Q^{k, \ell}, q^{k, \ell}\right) & =\mathcal{C}_{n, 1}^{+2}\left(q^{k, \ell}\right)+\mathcal{C}_{n, 1}^{-2}\left(q^{k, \ell}\right)+\mathcal{C}_{1, n}^{+2}\left(q^{k, \ell}\right) \\
& +\mathcal{C}_{1, n}^{-2}\left(q^{k, \ell}\right)+2 \delta_{Q^{k, \ell}, 0} \mathcal{C}_{1, n}^{+}\left(q^{k, \ell}\right) \mathcal{C}_{n, 1}^{+}\left(q^{k, \ell}\right) \\
G_{2}\left(Q^{k, \ell}, q^{k, \ell}\right) & =\mathcal{C}_{1, n}^{+2}\left(q^{k, \ell}\right)+\mathcal{C}_{1, n}^{-2}\left(q^{k, \ell}\right) \\
J_{1}\left(Q^{k, \ell}, q^{k, \ell}\right) & =\mathcal{C}_{n, 1}^{+2}\left(q^{k, \ell}+1\right)+\mathcal{C}_{n, 1}^{-2}\left(q^{k, \ell}+1\right) \\
& +\mathcal{C}_{1, n}^{+2}\left(q^{k, \ell}+1\right)+\mathcal{C}_{1, n}^{-2}\left(q^{k, \ell}+1\right) \\
& +2 \delta_{Q^{k, \ell}, 0} \mathcal{C}_{1, n}^{+}\left(q^{k, \ell}+1\right) \mathcal{C}_{n, 1}^{+}\left(q^{k, \ell}+1\right) \\
J_{2}\left(Q^{k, \ell}, q^{k, \ell}\right) & =\mathcal{C}_{1, n}^{+2}\left(q^{k, \ell}+1\right)+\mathcal{C}_{1, n}^{-2}\left(q^{k, \ell}+1\right)
\end{aligned}
$$

We also have $\kappa_{a}=\operatorname{cum}\left(a_{\ell}, a_{\ell}, a_{\ell}, a_{\ell}\right), M_{\ell}=\mathbb{E}_{\tau}\left[e^{-2 \tau_{\ell} / \gamma}\right]$, and $M_{\ell, k}=\mathbb{E}_{\tau}\left[e^{-\left(\tau_{\ell}+\tau_{k}\right) / \gamma}\right]$. Notice that closed-form expressions can be obtained for $M_{\ell}$ and $M_{\ell, k}$ which are omitted due to the lack of space.

In the sequel, we only focus on the derivations of $Y_{m}^{k, \ell}$ (with $k>\ell$ ). The other terms can be derived in a similar way. By noticing that $\tau_{k}+\tau_{\ell}=2 \tau_{\ell}+\Delta \tau_{k, \ell}$ and that $\tau_{\ell}$ is independent of $\Delta \tau_{k, \ell}$ as soon as $k>\ell$, we get

$$
Y_{m}^{k, \ell}=M_{\ell} X_{m}^{k, \ell}
$$

with

$$
\begin{aligned}
X_{m}^{k, \ell} & =\mathbb{E}_{\Delta \tau_{k, \ell}}\left[e^{-\Delta \tau_{k, \ell} / \gamma} G_{m}\left(Q^{k, \ell}, q^{k, \ell}\right)\right. \\
& \left.\times r^{2}\left(\Delta \tau_{k, \ell}-Q^{k, \ell} N_{f} T_{f}-q^{k, \ell} T_{c}\right)\right] .
\end{aligned}
$$

We would like to find a closed-form expression for $X_{m}^{k, \ell}$. Before going further, one can easily check that $\Delta \tau_{k, \ell}$ has the following probability density functions

$$
p_{\Delta \tau_{k, \ell}}(t)=\frac{\lambda^{k-\ell}}{(k-\ell-1) !} t^{k-\ell-1} e^{-\lambda t} \mathbf{1}_{t \geq 0} .
$$

Let $t:=\Delta \tau_{k, \ell}, Q_{t}=Q^{k, \ell}, q_{t}=q^{k, \ell}$ and $\varepsilon_{t}=\varepsilon^{k, \ell}$. By splitting the interval $[0,+\infty)$ into an infinite number of intervals of length $T_{c}$ and by using the fact that $Q_{t}=Q_{n N_{f} T_{f}}=Q$ for $t \in\left[Q N_{f} T_{f},(Q+1) N_{f} T_{f}\right)$ and $q_{t}=q_{n^{\prime} T_{c}}=q$ for $t \in\left[q T_{c},(q+1) T_{c}\right)$ with $Q$ and $q$ two integers, one can see that $X_{m}^{k, \ell}$ takes the following form

$$
\begin{aligned}
X_{m}^{k, \ell} & =\frac{\lambda^{k-\ell}}{(k-\ell-1) !} \sum_{q=0}^{N_{c} N_{f}-1} \sum_{Q=0}^{+\infty} G_{m}(Q, q) \\
& \times e^{-(\lambda+1 / \gamma)\left(Q N_{f} T_{f}+q T_{c}\right)} \\
& \times \int_{0}^{T_{c}}\left(Q N_{f} T_{f}+q T_{c}+\varepsilon\right)^{k-\ell-1} e^{-(\lambda+1 / \gamma) \varepsilon} r^{2}(\varepsilon) d \varepsilon .
\end{aligned}
$$

Merging the previous equation and Eq. (6) into Eq. (5) leads to the final result

$$
\begin{aligned}
V & =\sum_{n=2}^{K}\left(\sum _ { \ell = 1 } ^ { L _ { r } } \sum _ { k = 1 } ^ { L _ { r } } \left(\left(\kappa_{a} M_{\ell} \delta_{k, 1}+\sigma_{a}^{4} M_{\ell, k}\right)\left(\mathcal{C}_{1, n}^{+2}(0)+\mathcal{C}_{1, n}^{-2}(0)\right)\right.\right. \\
& \left.+\sigma_{a}^{4} M_{\ell}\left(\mathcal{C}_{1, n}^{+2}(0)+\mathcal{C}_{1, n}^{-2}(0)+\mathcal{C}_{1, n}^{+}(0) \mathcal{C}_{n, 1}^{+}(0)\right)\right) r^{2}(0) \\
& +\sigma_{a}^{4} \sum_{Q=0}^{+\infty} \sum_{q=0}^{N_{c} N_{f}-1}\left(\mathcal{C}_{n, 1}^{+2}(q)+\mathcal{C}_{n, 1}^{-2}(q)+\mathcal{C}_{1, n}^{+2}(q)\right. \\
& \left.+\mathcal{C}_{1, n}^{-2}(q)+2 \delta_{Q, 0} \mathcal{C}_{1, n}^{+}(q) \mathcal{C}_{n, 1}^{+}(q)\right) \Phi_{1}(Q, q) \\
& +\sigma_{a}^{4} \sum_{Q=0}^{+\infty} \sum_{q=0}^{N_{c} N_{f}-1}\left(\mathcal{C}_{1, n}^{+2}(q)+\mathcal{C}_{1, n}^{-2}(q)\right) \Psi_{1}(Q, q) \\
& +\sigma_{a}^{4} \sum_{Q=0}^{+\infty} \sum_{q=0}^{N_{c} N_{f}-1}\left(\mathcal{C}_{n, 1}^{+2}(q+1)+\mathcal{C}_{n, 1}^{-2}(q+1)+\mathcal{C}_{1, n}^{+2}(q+1)\right. \\
& \left.+\mathcal{C}_{1, n}^{-2}(q+1)+2 \delta_{Q, 0} \mathcal{C}_{1, n}^{+}(q+1) \mathcal{C}_{n, 1}^{+}(q+1)\right) \Phi_{2}(Q, q) \\
& \left.+\sigma_{a}^{4} \sum_{Q=0}^{+\infty} \sum_{q=0}^{N_{c} N_{f}-1}\left(\mathcal{C}_{1, n}^{+2}(q+1)+\mathcal{C}_{1, n}^{-2}(q+1)\right) \Psi_{2}(Q, q)\right)
\end{aligned}
$$

with

$$
\begin{gathered}
\Phi_{1}(Q, q)=\phi(Q, q) \sum_{\ell=1}^{L_{r}} \frac{1}{(\lambda+2 / \gamma)^{\ell}} \sum_{k=\ell+1}^{L_{r}} \frac{\lambda^{k}}{(k-\ell-1) !} \\
\times \int_{0}^{T_{c}}\left(Q N_{f} T_{f}+q T_{c}+\varepsilon\right)^{k-\ell-1} e^{-(\lambda+1 / \gamma) \varepsilon} r^{2}(\varepsilon) \mathrm{d} \varepsilon \\
\Phi_{2}(Q, q)=\phi(Q, q) \sum_{\ell=1}^{L_{r}} \frac{1}{(\lambda+2 / \gamma)^{\ell}} \sum_{k=L_{r}+1}^{N_{p}} \frac{\lambda^{k}}{(k-\ell-1) !} \\
\times \int_{0}^{T_{c}}\left(Q N_{f} T_{f}+q T_{c}+\varepsilon\right)^{k-\ell-1} e^{-(\lambda+1 / \gamma) \varepsilon} r^{2}(\varepsilon) \mathrm{d} \varepsilon \\
\Psi_{1}(Q, q)=\phi(Q, q) \sum_{\ell=1}^{L_{r}} \frac{1}{(\lambda+2 / \gamma)^{\ell}} \sum_{k=\ell+1}^{L_{r}} \frac{\lambda^{k}}{(k-\ell-1) !} \\
\times \int_{0}^{T_{c}}\left(Q N_{f} T_{f}+q T_{c}+\varepsilon\right)^{k-\ell-1} e^{-(\lambda+1 / \gamma) \varepsilon} r^{2}\left(\varepsilon-T_{c}\right) \mathrm{d} \varepsilon
\end{gathered}
$$




$$
\begin{aligned}
& \Psi_{2}(Q, q)=\phi(Q, q) \sum_{\ell=1}^{L_{r}} \frac{1}{(\lambda+2 / \gamma)^{\ell}} \sum_{k=L_{r}+1}^{N_{p}} \frac{\lambda^{k}}{(k-\ell-1) !} \\
& \times \int_{0}^{T_{c}}\left(Q N_{f} T_{f}+q T_{c}+\varepsilon\right)^{k-\ell-1} e^{-(\lambda+1 / \gamma) \varepsilon} r^{2}\left(\varepsilon-T_{c}\right) \mathrm{d} \varepsilon
\end{aligned}
$$

and

$$
\phi(Q, q)=e^{-(\lambda+1 / \gamma)\left(Q N_{f} T_{f}+q T_{c}\right)} .
$$

The rigorous analysis of the closed-form expression $V$ versus various parameters (time-hopping code, channel parameters, number of fingers) is not easy. Nevertheless computing this theoretical (but complicated) expression is much faster than computing the empirical multicode variance of a complete UWB system via Monte-Carlo simulation. Therefore, even if this expression does not highlight the influence of the various above-mentioned parameters, it is of great interest.

In the case of a full rake receiver $\left(L_{r}=N_{p}\right)$, one can remark that $\Phi_{2}(Q, q)$ and $\Psi_{2}(Q, q)$ vanish, so the final expression of $V$ simplifies accordingly.

The expression of $V$ can be further simplified by averaging it over the time-hopping codes. We can assume that each vector $c_{n}(j)$ is the realization of an i.i.d. random vector whose components admit, each, the following distribution $p(c)=$ $\left(\left(N_{c}-1\right) \delta(c)+\delta(c-1)\right) / N_{c}$. According to such a distribution, we obtain that

$$
\begin{aligned}
\mathbb{E}_{c}\left[\mathcal{C}_{1, n}^{+2}(q)\right] & =\frac{\left(N_{c} N_{f}-q\right)^{2}+\left(N_{c} N_{f}-q\right)\left(N_{c}^{2}-1\right)}{N_{c}^{4}} \\
\mathbb{E}_{c}\left[\mathcal{C}_{1, n}^{-2}(q)\right] & =\frac{q^{2}+q\left(N_{c}^{2}-1\right)}{N_{c}^{4}} \\
\mathbb{E}_{c}\left[\mathcal{C}_{1, n}^{-}(q) \mathcal{C}_{n, 1}^{-}(q)\right] & =\frac{q^{2}}{N_{c}^{4}} \\
\mathbb{E}_{c}\left[\mathcal{C}_{1, n}^{+}(q) \mathcal{C}_{n, 1}^{+}(q)\right] & = \begin{cases}\frac{N_{f}\left(N_{c} N_{f}-1\right)+N_{c}^{2} N_{f}}{N_{c}^{3}} & q=0 \\
\frac{\left(N_{c} N_{f}-q\right)^{2}}{N_{c}^{4}} & q \neq 0 .\end{cases}
\end{aligned}
$$

when $n \neq 1$. Although these above closed-form expressions do not take into account the frame structure of the UWB signal, they are numerically in agreement with their empirical values when the frame structure of the UWB signal is considered.

Let $U:=\mathbb{E}_{a, \tau, d}\left[z_{u}^{2}\right]$ denote the energy of the useful part of the received signal at the rake receiver output. $U$ can be deduced from $V$ by removing the sum on $n$ and by putting $n=1$.

\section{NumERICAL ILLUSTRATIONS}

The UWB-IR system is obtained by setting $L_{r}=3, N_{p}=3$, and $T_{c}=5 \mathrm{~ns}$. The other parameters vary accordingly to the analysis we want to show. Their value will be given when necessary. The pulse $w(t)$ is designed such that its spectrum fits well the shape of the FCC spectral mask [9]. For practical purposes, the pulse (with unitary energy) is truncated with the duration $T_{w}=1 \mathrm{~ns}$. For the sake of simplicty, we choose $L_{r}=N_{p}$, i.e. a full rake receiver.

In order to validate our approach, we display in Fig. 1 a comparison between a set of different Monte Carlo simulations and their equivalent analytical expressions, for different sets of parameters, as a function of the number of allocated codes. For each point of the curve, 10,000 trials are run for the empirical values of the MCI variance. The statistical parameters $\lambda$ and $\gamma$ were chosen to match those of [5] and [6].

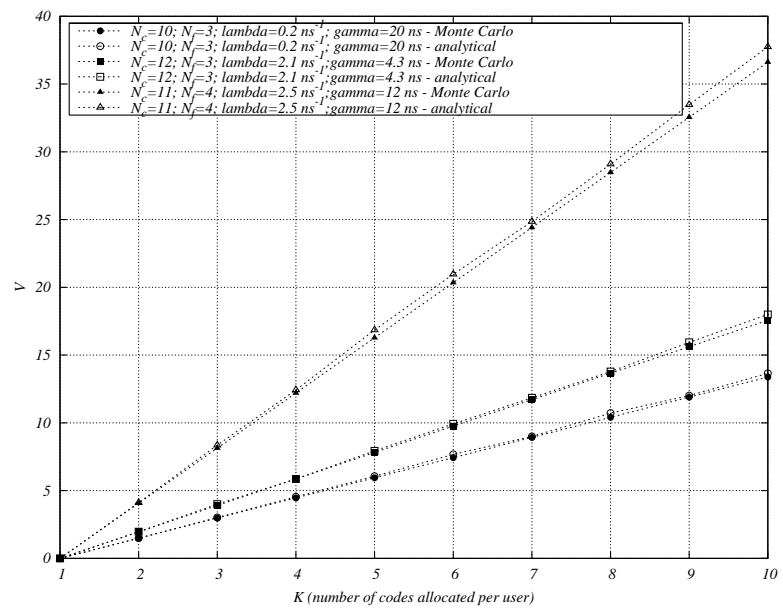

Fig. 1. Empirical and theoretical MCI variance versus number of codes.

Now, with the analytical expression of the MCI variance in hand, we can easily study its behavior with respect to different parameters of the system and the propagation channel. In Figs. 2 and 3 we show various curves for $V$ versus $\gamma$ and $\lambda$, respectively. We set $N_{f}=3, N_{c}=10$ and, since we know that $V$ varies linearly $K$, we fix this value to 2 .

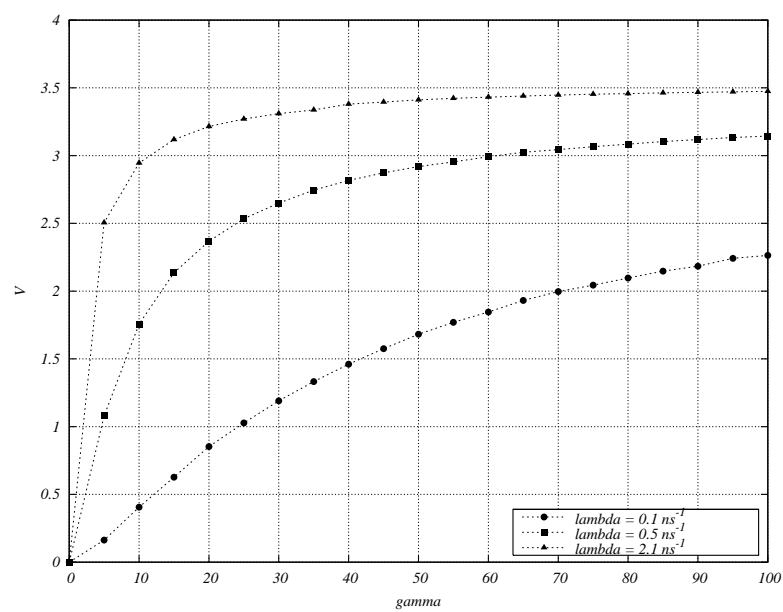

Fig. 2. $\quad V$ versus $\gamma$.

As we can remark from Figs. 2 and 3, the MCI variance increases with $\lambda$ and $\gamma$. These parameters are associated with delay density and channel length, respectively. In other words, long channels that decay slowly and channels with high delay densities lead to important multicode interferences. We also note that the interference is more sensible to variations in the channel's statistics for small parameters values, especially $\lambda$.

In Fig. 4, we show the influence of the UWB signal structure on the MCI. For this, we fix the length of the signal and vary $N_{c}$ and $N_{f}$ individually. As expected, as $N_{f}$ increases and $N_{c}$ decreases, the MCI becomes more important, with a steeper 


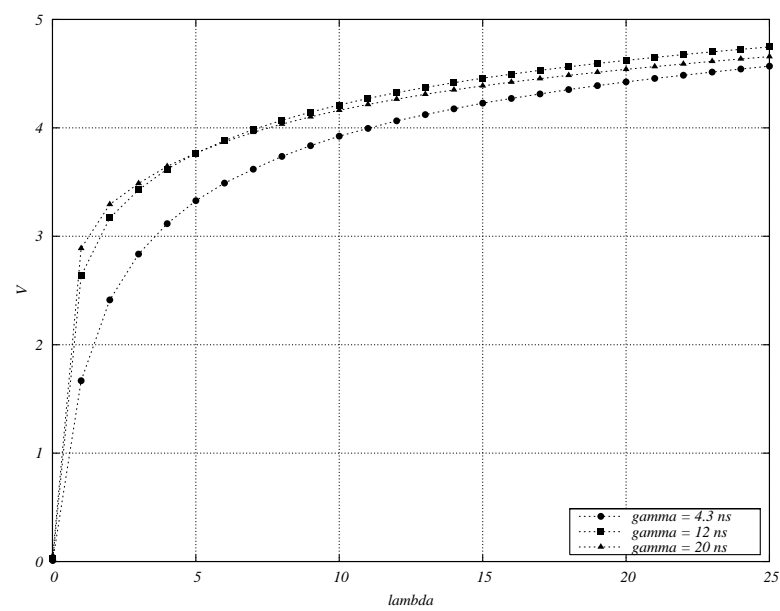

Fig. 3. $V$ versus $\lambda$.

inclination at each step in $N_{f}$. This can be explained by the fact that the codes are not orthogonal and, as $N_{c}$ becomes smaller, the number of collisions becomes bigger.

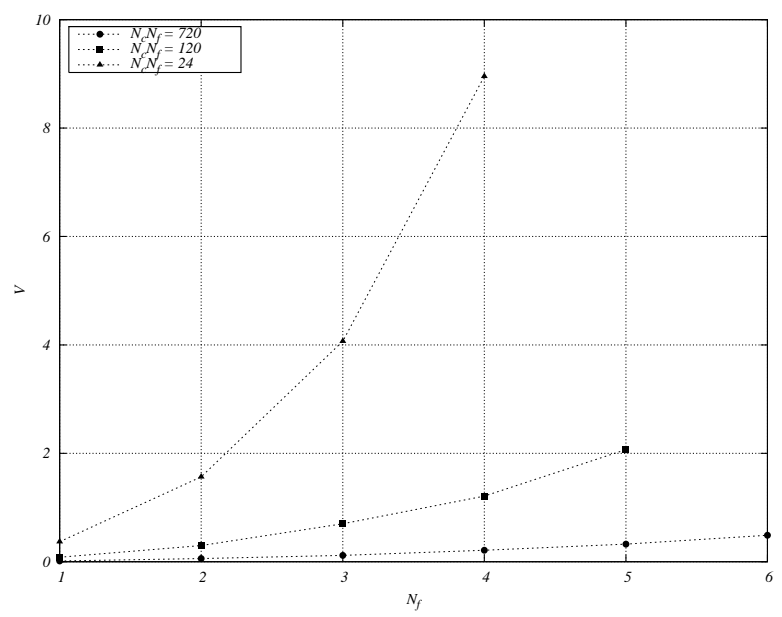

Fig. 4. $\quad V$ versus $N_{f}$.

Another important analysis within this context concerns the system's maximum data rate. Indeed, the analytical expression of $V$ can give us an efficient way to roughly estimate the maximum data rate. This can come in extremely handy in scenarios where fast decisions must be made on resource allocation. Thus, under the assumption that $V$ is gaussian, the ergodic maximum rate can be defined as

$$
R_{\max }^{(e)}=\mathbb{E}_{a, \tau, c}\left[K \log _{2}\left(1+\frac{z_{u}^{2}}{z_{m c i}^{2}+\sigma_{\eta}^{2}}\right)\right],
$$

which can be further approximated by

$$
R_{m a x}^{(a)}=K \log _{2}\left(1+\frac{U}{V+\mathbb{E}_{a, \tau}\left[\sigma_{\eta}^{2}\right]}\right) .
$$

Fig. 5 compares ergodic and approximate maximum data rates, as functions of the number of codes, for different system parameters.

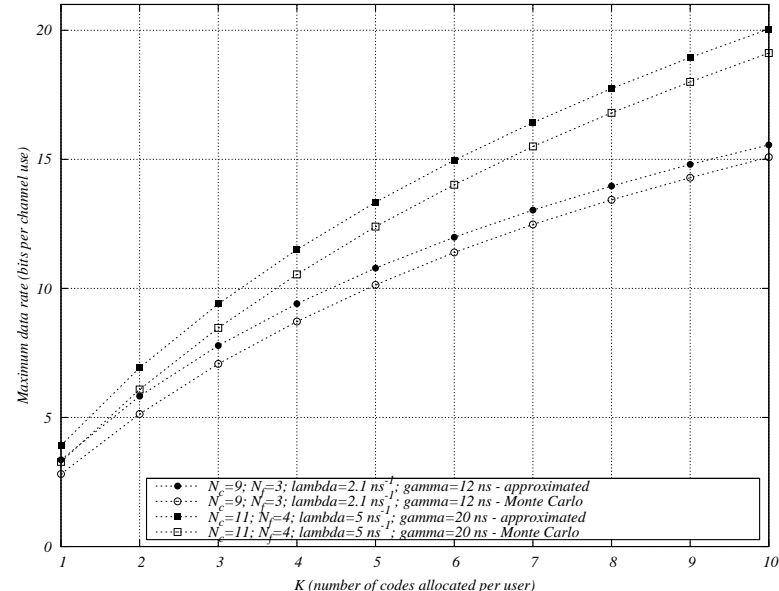

Fig. 5. Maximum data rate versus $K$.

\section{CONCLUSION}

In this paper, we derived a closed form expression for the multicode interference variance in an IR-UWB system, that allowed us to highlight the influence of the different channel and system parameters. We were also able to provide a rough estimate of the achievable data rates of the system. Future investigations may include a more accurate analysis of the expression of $V$, a comparative study with respect to timehopping codes and an introduction of multicodes detection to improve performance, at the expense of complexity.

\section{REFERENCES}

[1] M. Z. Win, and R. A. Scholtz, "Ultra-wide bandwidth time-hopping spread-spectrum impulse radio for wireless multiple-access communications," IEEE Trans. on Communications, vol. 48, no. 4, pp. 679-691, April 2000.

[2] D. Cassioli, M. Z. Win, F. Vatalaro and A. F. Molish, "Performance of low-complexity rake reception in a realistic UWB Channel," in Proc. of IEEE International Conference on Communications, New York (USA), May 2002.

[3] C.J. Le Martret, A.-L. Deleuze, and P. Ciblat, "Optimal Time-Hopping Code Criterion for Multi-User Interference Mitigation in Ultra-Wide Bandwidth Impulse Radio," IEEE Trans. on Wireless Communications, vol. 5, no. 6, pp. 1516-1525, Mar. 2005.

[4] A.-L. Deleuze, P. Ciblat, and C.J. Le Martret, "Inter-Symbol/Inter-Frame Interference in Time-Hopping Ultra Wideband Impulse Radio system," in Proc. of IEEE International Conference on Ultra-Wideband (ICU), Zürich (Switzerland), Sept. 2005.

[5] A.A.M. Saleh, and R.A. Valenzuela, "A statistical model for indoor multipath propagation," IEEE Journal on Selected Areas in Communications, vol. 5, no. 2, pp. 128-137, Feb. 1987.

[6] A.F. Molish, J.R. Foerster, and M. Pendergrass, "Channel models for ultrawideband personal area networks," IEEE Wireless Communications, vol. 10, no. 6, pp. 14-21, Dec. 2003.

[7] A.-L. Deleuze, P. Ciblat, and C.J. Le Martret, "Rake receiver improvement for residual interference cancellation in UWB context," in Proc. of IEEE Vehicular Technology Conference (VTC), Dublin (Irland), April 2007.

[8] M. Eslami, X. Dong, "Rake-MMSE equalizer performance for UWB," IEEE Communications Letters, vol. 9, no. 6, pp. 502-504, June 2005.

[9] Federal Communication Commission, "Revision of Part 15 of the commission's rules regarding ultra-wideband transmission systems, first Report and Order," FCC, Feb. 2002. 